possible that this is a good technique and yields a high percentage of successful results, and since its effects are carried throughout the lymphatic system, approaches the ideal of a basic treatment in the same way as diphtheria antitoxin is a basic treatment. I will now demonstrate on a case the selection of areas, the dosage, and method of application.

Conclusion.-In conclusion, may I repeat that I have warned you against the disappointments that will follow if you employ electrical currents without a sound physiological reason for their employment, or because you want to do something for the patient. A new method and apparatus for diagnosis has been demonstrated and cases shown illustrative of the importance of basic diagnoses in the treatment of rheumatism. Finally, the majority of our successful results at the British Red Cross Clinic for Rheumatism come from the fact that the patient as a whole entity is sent to us for consultation and advice, and for the prescription of such treatment, medicinal, dietetic, or physical, as we think best. $\mathrm{He}$ is not sent to us with a request for an electrical current to a particular site.

\title{
ACUTE THYREOIDITIS.
}

\section{By ROLAND T. de HELLEBRANTH, M.D.}

Ventnor City, N.J.

Although almost every infectious disease is likely to be the source of acute or chronic thyreoiditis, in the experience of the majority of surgeons this clinical entity is located by them more trequently in textbooks than in everyday practice.

Thyreoiditis is rarer than strumitis, for the gland is seldom subject to inflammation, and a termination in suppuration is also rare indeed. In acute infections, a light inflammation of the thyreoid is often an accessory sign, particularly in the early stages of syphilis, in more than half these cases. Instances of severe alcoholism and tuberculosis show slight sclerotic processes, but more severe inflammatory changes are observable after attacks of malaria, influenza, typhoid, \&c., and particularly following acute articular rheumatism and angina. Demme and Brisson report even epidemics of thyreoiditis having followed measles.

Acute thyreoiditis which dots not go on to suppuration has a sudden onset, with general symptoms and fever. The gland swells, local symptoms of pressure arise, and severe pains begin to radiate to the ear and the throat. Usually after a quickly arriving climax there is a subsidence of all these phenomena.

Author's Case Report.-A 27-year-old woman, mother of one child, complained of severe pain in her neck on attempting to swallow. She gave no previous history, until questioning evoked the fact that one month previous, suffering from constipation, she strained herself and felt a sudden sensation as though something had burst in her neck.

A few weeks later she had a mild tonsillitis, and it was then that she began to feel a slight tenderness in her neck. She did not pay much attention to it until one morning she woke up unable to swallow.

On examination an extremely sensitive and painful swelling was found between 
the hyoid bone and the jugular fossa about the size of a walnut, this following promptly the movements of the larynx. The contour of the thyreoid gland was prominent in its circumference, and the entire gland was slightly enlarged and very tender on palpation. Temperature was $100 \frac{2}{6}{ }^{\circ} \mathrm{F}$., and the pulse-rate Iro.

Diagnosis of acute thyreoiditis with circumscribed abscess was made. A small horizontal incision was then made through the skin in one of the wrinkles at the usual site for thyreoidectomy. The fibres of the platysma, sternothyreoid and sternohyoid muscles were bluntly dissected and the abscess opened. It contained I $_{5}$ c.c. purulent discharge, the smear of which showed a great count of lymphocytes and innumerable staphylococci. The cavity of the abscess seemed to be pear-shaped and completely isolated from the surrounding tissues. The patient's difficulties with swallowing ceased almost at once, and the same evening her temperature became normal. The abscess was kept open for four days by means of a small strip of iodoform gauze. When the discharge became scanty, the drain was removed and the wound closed spontaneously the eighth day. The contour of the thyreoid gland disappeared and all tenderness subsided rapidly.

Similar Cases.-In 1904, Henry Roth recognized acute suppurative thyreoiditis as a very rare condition, which usually was secondary to some infectious disease. He reported what he considered a primary infection. When the enlarged gland was incised, a few drops of pus escaped. Areas of necrosis were shown after operation and the patient died the next day. Infection was due to streptococci, but the cause could not be determined. The right lobe was more involved than the other side, symptoms of sepsis being pre-eminent. Though the local signs might have been those of an ordinary phlegmone, the constitutional symptoms were too severe.

Two more cases of suppurative thyreoiditis were reported by L. Rogers which exhibited an almost complete lack of symptoms. Both patients appeared exceptionally healthy, there being only slight discomfort. On surface examination little could be found, and it required roentgen-ray and laboratory diagnosis to make out the cases.

Acute attacks occur extremely rarely in infancy. The patient, a young child, could not move its head, the eyes being congested, the lips cyanotic, and palpation of the swelling on the neck caused pain. The observations of Baratta were to the effect that it was an acute case due to its rapid evolution and lack of preceding findings. Suffocation symptoms subsided after two punctures were made with removal of 25 c.c. and of 20 c.c. of pus. Use of compresses with solution of boric and salicylic acids brought relief and cure. In one of Baratta's cases, swelling was noted in the anterior and medial part of the neck, the longer axis of the swelling being in a transverse direction. Laterally it continued on and merged with the sternocleidomastoid muscle. High up, on the level of the hyoid bone, the swelling stopped suddenly and a furrow distinctly separated it from the suprahyoid region. As in the author's case, the face wore an anxious look, and passive movements provoked pain. Respiration was rapid. During inspiration a prolonged sound like laryngeal stridor was noted.

Comment.-As was said before, among the sources of the acute or chronic thyreoiditis are almost all the infectious diseases, such as scarlet and typhoid fever, measles, puerperal fever, malaria, as also bronchitis, pneumonia, tonsillitis, syphilis, inflammatory rheumatism, influenza, erysipelas, \&c. Such thyreoiditis is due to bacterial toxins, but there are cases developing after extensive use of iodine, when the gland becomes inflamed. 
In experiments made on animals, thyreoiditis was produced by lions on injecting paraffin or calcium chlorite in the thyreoid gland. In the purulent forms, Simmonds, found great herds of streptococci and typhoid bacilli, but most frequently staphylococci, which may cause the general enlargement and diffuse inflammation of the thyreoid gland or yield a circumscribed abscess. The inciting agent may come in the form of a germembolus, either from the immediately surrounding tissues, through direct contact, or from distant parts of the body through the lymph or blond-stream.

Classification.-As far as types of thyreoiditis are concerned, it is possible to distinguish acute and chronic suppurative, acute and chronic non-suppurative, and woody thyreoiditis. Both the suppurative and non-suppurative thyreoiditis may cause only a slight tenderness and enlargement of the thyreoid gland, disappearing unnoticed, or better, undiagnosed, in eight or ten days. If they form an abscess, it may break through the skin or into the trachea, or it may heal up by fibrous atrophy, becoming the cause of a goitre. This atrophy may involve such a large part of the thyreoid gland as to lead to myxœdema.

The rarest type is the woody thyreoiditis, which usually produces symptoms of malignant neoplasm. It is hard to the touch and causes pain and dyspnœa.

All the forms of thyreoiditis mentioned affect mostly middle-aged women ; children and older people usually escape attack.

Microscopically, we find the capillary vessels enlarged, a hæmorrhage of which may readily occur. The lobules show leucocytic infiltration, and the acini contain a granular serum precipitable with a diminution of the colloid. The tension may subside or it may spread, or may form an isolated abscess. Dutrow and others have studied cases of acute idiopathic thyreoiditis or inflammation of the gland. Dutrow's case was accompanied by a leucocytosis of 12,000 .

The Etiology in our case is not very doubtful, although according to Wilhelm Falta nothing is known as to the causative agent. Most likely the strain or tension started the hæmorrhage into the glandular tissue, producing thereby the best culture medium for the staphylococci that caused the slight tonsillitis.

\section{BIBLIOGRAPHY.}

Baratta, A. Pediatria Naples, xxxvi, 602, June 1, 1928.

Brenizer, Addison G. Southern Surg. Assoc., Proceedings, December 14, 1928.

BRUCE, H. S. " Reidel's Tumour," Case, Ann. Surgery, xciv, 562, October, 1931

Demme and Brisson. Quoted by W. F. Reinhoff, Junr.

Dutrow, H. V. "Idiopathic Non-purulent Acute Thyreoiditis," Journ. Amer, Med. Assoc., 1911, p. 1761.

Falta, Wilhelm. Edited by Meyers, "Endocrine Diseases," Blackiston, p. 151.

"Herausgegeben von Ludwig Aschoff," Pathologische Anatomie.

KaPPIS, Prof. Dr. M. Chirurgische Diagnostik.

MaYo, C. H. "Diseases of the Thyreoid," Journ. Mich. Med. Society, xi, 1, January, 1912.

MoRA, J. M. "Acute Suppurative Thyreoiditis in Childhood" : Operative Recovery, Amer. Journ. Dis. Child., xl, 500-2, September.

Roth, Henry. "Acute Thyreoiditis," Amer. Journ. Med. Sciences, January, 1904.

Rothe, F. A. "Non-suppurative Acute Thyreoiditis," Surg. Clin. in N. Amer., xi, 1449-54, December, 1931.

Rogers, L. Lancet, i, 866, April 23, 1927. 